\title{
First Results From The Balloon Flight Of The NCT Prototype
}

\author{
Wayne Coburn $^{a}$, Steven E. Boggs ${ }^{a, b}$, Jason D. Bowen ${ }^{a}$, Mark E. Bandstra ${ }^{a}$, \\ Mark S. Amman ${ }^{c}$, Morgan T. Burks ${ }^{e}$, William Craig ${ }^{e}$, Pierre Jean ${ }^{d}$, Robert P. Lin ${ }^{a, b}$, Paul N. \\ Luke $^{c}$, Norman W. Madden ${ }^{e}$, David M. Smith ${ }^{a}$, Peter von Ballmoos ${ }^{d}$ \\ ${ }^{a}$ Space Sciences Laboratory, University of California, Berkeley, CA 94720, USA \\ ${ }^{b}$ Department of Physics, University of California, Berkeley, CA 94720, USA \\ ${ }^{c}$ Lawrence Berkeley National Laboratory, University of California, Berkeley, CA 94720, USA \\ ${ }^{d}$ Centre d'Etude Spatiale des Rayonnements, CNRS/UPS, BP 4346, 31029 Toulouse Cedex, France \\ ${ }^{e}$ Lawrence Livermore National Laboratory, Livermore, CA 94550, USA
}

\begin{abstract}
We flew a prototype of the Nuclear Compton Telescope (NCT) on a high altitude balloon from Fort Sumner, New Mexico on 2005 June 1. The NCT prototype is a soft $\gamma$-ray $(0.2-15 \mathrm{MeV})$ telescope designed to study, through spectroscopy, imaging, and timing, astrophysical sources of nuclear line emission and $\gamma$-ray polarization. Our program is designed to develop and test the technologies and analysis techniques crucial for the Advanced Compton Telescope satellite, while studying $\gamma$-ray radiation with very high spectral resolution, moderate angular resolution, and high sensitivity. The NCT prototype utilizes two, 3D imaging germanium detectors (GeDs) in a novel, ultra-compact design optimized for nuclear line emission $(0.5-2 \mathrm{MeV})$ and polarization in the $0.2-0.5 \mathrm{MeV}$ range. Our prototype flight was a critical test of the novel instrument technologies, analysis techniques, and background rejection procedures we have developed for high resolution Compton telescopes.
\end{abstract}

Keywords: Germanium detectors; gamma-ray spectroscopy; gamma-ray astronomy; gamma-ray polarization; balloon payloads

\section{INTRODUCTION}

The Nuclear Compton Telescope (NCT) is a balloonborne soft $\gamma$-ray $(0.2-15 \mathrm{MeV})$ telescope designed to study astrophysical sources of nuclear line emission and $\gamma$-ray polarization $(1 ; 2)$. It employs a novel Compton telescope design (fig. 1), utilizing twelve high spectral resolution germanium detectors (GeDs) with the ability to track the location in three dimensions of each photon interaction. Tracking serves three purposes: imaging the sky using Compton imaging techniques, measuring polarization, and very effectively reducing background.

\footnotetext{
Further author information:

E-mail: wcoburn@ssl.berkeley.edu, Telephone: +1-510-6431855
}

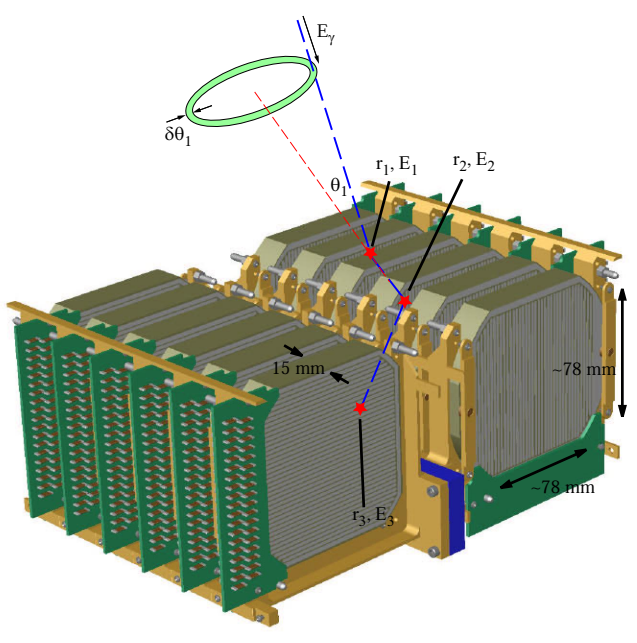

Figure 1. The heart of NCT is an array of 12 cross-strip GeDs with 3-D position resolution, excellent spectroscopy, sensitivity to $\gamma$-ray polarization, and high efficiency. The source direction is upwards in this cartoon, with the detectors illuminated edge on.

The entire set of detectors and their cryostat are enclosed inside an active BGO well (fig. 2), giving an overall field of view of $3.2 \mathrm{str}$. The instrument is mounted in a pointed, autonomous balloon platform and is capable of long duration ( $\sim 2$ week) balloon flights. NCT is designed to optimize sensitivity to nuclear line emission over the crucial $0.5-2 \mathrm{MeV}$ range, and sensitivity to polarization in the $0.2-0.5 \mathrm{MeV}$ range. The guiding principle of NCT is that high efficiency and excellent background reduction are critical for advances in soft $\gamma$-ray sensitivity.

The sensitivity of NCT should be signal-limited, not background limited for most astrophysical sources. This means that NCT will explore a new phase space of nuclear $\gamma$-ray observations. Some of the long term goals of NCT include mapping both ${ }^{26} \mathrm{Al}$ and ${ }^{60} \mathrm{Fe}$ in the plane and bulge of our galaxy, measuring the amount of

UV, X-Ray, and Gamma-Ray Space Instrumentation for Astronomy XIV edited by Oswald H. W. Siegmund, Proceedings of SPIE Vol. 5898 589802, (2005) · 0277-786X/05/\$15 - doi: 10.1117/12.615876 


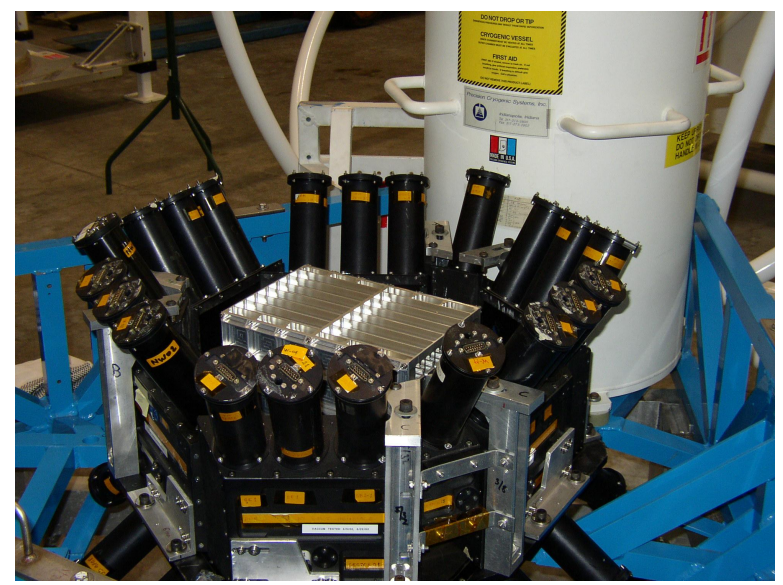

Figure 2. NCT flight cryostat during integration tests with the BGO shields into the instrument cradle. The cradle assembly, including shields and dewar, can pivot to allow pointing at an arbitrary positive elevation.

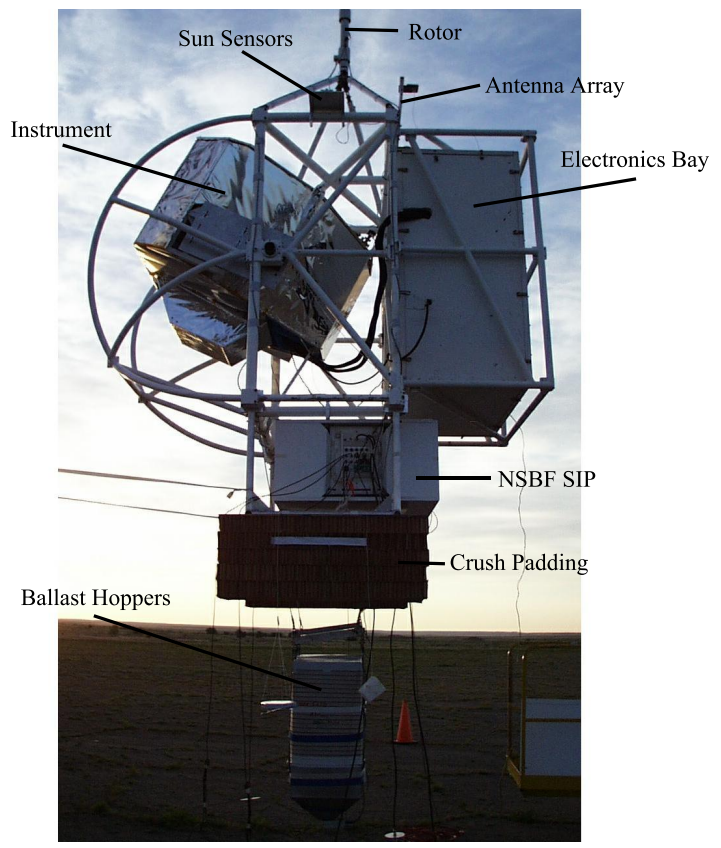

Figure 3. Picture of the gondola frame in profile, showing the relative positions of the instrument, electronics bay, SIP, and ballast hoppers.

${ }^{44} \mathrm{Ti}$ contained in recent core collapse supernova remnants, measuring with high resolution the spectra of AGN and searching for $\gamma$-ray polarization, looking for polarization in the emission from pulsars and associated plerions, and searching for redshifted deuterium lines from the surfaces of neutron stars.

The NCT prototype is a scaled down, two detector version of NCT. The NCT prototype flew on a high al-

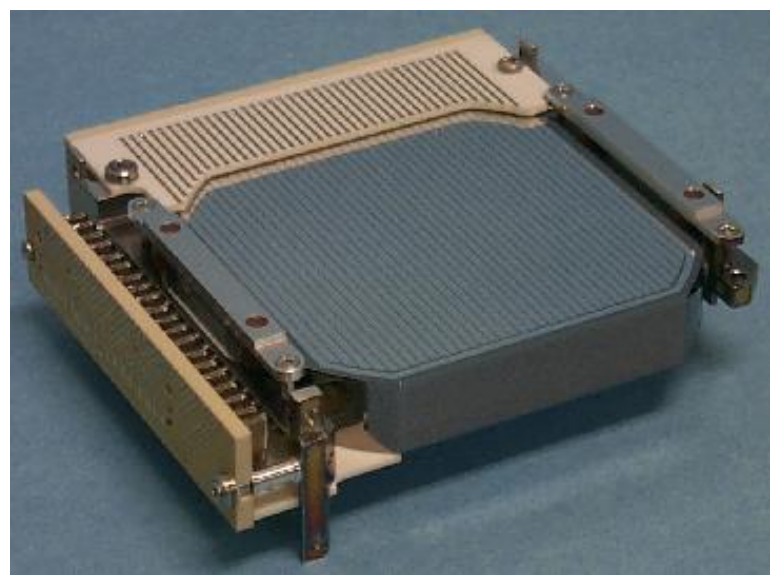

Figure 4. Photograph of one of our two flight detectors. Each detector has $37 \times 37$ strips, with an active volume of $82.1 \mathrm{~cm}^{3}$ and containing over 41 -thousand pixel-volume elements.

titude balloon from Fort Sumner, New Mexico on 2005 June 1. The duration of the flight was approximately nine hours.

We had three main goals for the NCT prototype balloon flight. Our primary goal was to qualify the gondola for a future, Long Duration Balloon Flight (LDBF) from Alice Springs, Australia. This was accomplished. Our secondary goal was to measure our instrumental background, along with our telemetry and data vault needs, at balloon altitudes. This was also accomplished. Our tertiary goal was to obtain images and spectra of the Crab Nebula/Pulsar and possibly the transient Xray pulsar $1 \mathrm{~A} 0535+262$. The analysis of the data from the flight is ongoing, with results expected soon $(3 ; 4)$.

The NCT gondola frame is $\sim 7 \times 9 \mathrm{ft}$ wide, and with the NASA electronics package (SIP) and ballast hoppers it is over $\sim 20 \mathrm{ft}$ tall. The total weight of the NCT prototype, minus ballast and SIP, is $2370 \mathrm{lbs}$. For the flight we had an additional $1200 \mathrm{lbs}$ of non-magnetic (sand) ballast. Fig. 3 is a picture of the gondola frame as seen from the side, and shows the locations of the various components discussed in detail below.

\section{INSTRUMENTATION}

At the heart of the NCT prototype are two large volume, 3-D positioning cross strip GeDs, fabricated at LBNL using their amorphous Ge contact technology (5). Each $\mathrm{GeD}$ is a $37 \times 37$ strip $15 \mathrm{~mm}$ thick p-type planar detector. Orthogonal strips were deposited on both faces of the $\mathrm{GeD}$, with a strip pitch of $2 \mathrm{~mm}$ and a $0.25 \mathrm{~mm}$ gap between strips. A $2 \mathrm{~mm}$ thick guard ring 


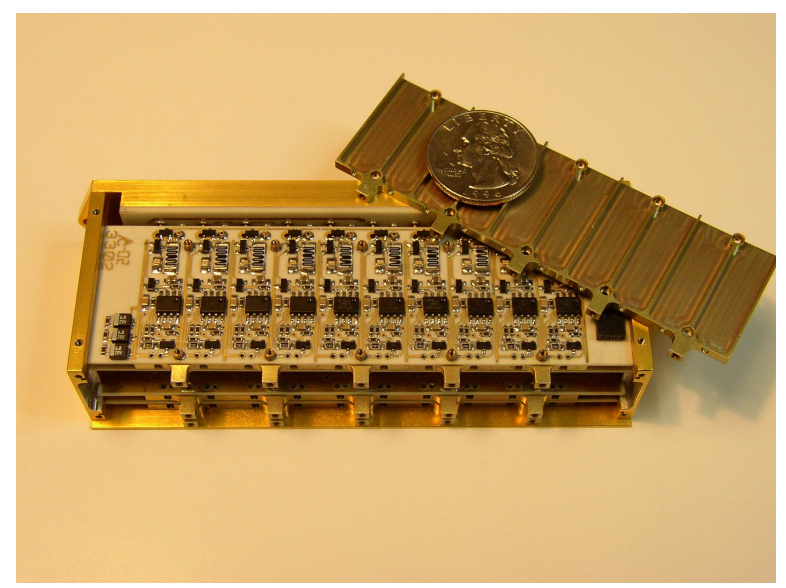

Figure 5. NCT flight preamps in their shielded housing, with 10 compact preamps per board, four boards per GeD face. (The top cover has been removed and flipped to show how each preamp is electrically shielded for low noise.)

surrounds this active area on both faces of the detector, with a $1 \mathrm{~mm}$ gap between the ring and the edge of the crystal. We instrumented the strips on both the ground (DC coupled, anode side) and HV (AC coupled, cathode side) sides with custom low power, low noise preamplifiers (6).

In the $12-\mathrm{GeD}$ design for $\mathrm{NCT}$, the detectors are illuminated edge on rather than face on (fig. 1). We did this to maximize our effective area at soft $\gamma$-ray energies. For the NCT prototype, which has only two detectors, this edge-on configuration is not optimal. Therefore we opted to remove several shield pieces and rotated the detector assembly so that the side of the cryostat was exposed to our sources. In this orientation, the detectors were closer to face-on than edge-on to the sky. This increased our effective area, although it meant that detector 1 was behind and partially shadowed by detector 0 .

NCT uses custom GeD quality signal processing electronics. Each detector strip has a compact, low power signal processing chain made predominantly of conventional surface mount components. Detector signal extraction is accomplished with a unique charge sensitive preamplifier (fig. 5), in which excellent spectroscopic performance is achieved in a small footprint and at modest cost and low power, without sacrificing signal bandwidth (7). A much-simplified pulse-shaping amplifier, with both a fast and a slow channel, follows each preamplifier. The slow channel, with a $3 \mu$ s peaking time, is followed by a peak detect and stretch function. The fast channel uses a small delay line constant fraction discriminator to time stamp each waveform at $50 \%$

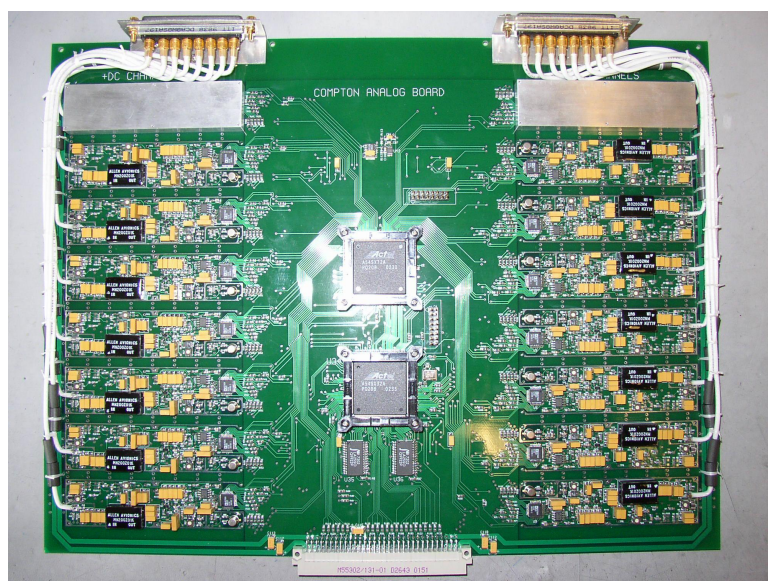

Figure 6. NCT signal processing (or "analog") board, containing 16 detector channels. Electric shield covers are removed from all but the top two channels. Each analog board utilizes two ACTELs sitting at the center of the board. The first ACTEL is for coincidence logic, and the second for collecting rates in each channel. A cluster of five analog boards is required to fully instrument each detector.

of its maximum amplitude, generating a low time walk signal. Demonstrated resolutions are $1.2 \mathrm{keV}$ FWHM at $60 \mathrm{keV}$, and $0.5 \mathrm{~mm}$ FWHM depth determination for all interactions that deposit $\geq 40 \mathrm{keV}$. Spectroscopy signals uniquely match the fast-signal pairs for multiple interactions in the same GeD.

One 16-channel signal-processing cluster resides on a single printed circuit card (fig. 6), with both the fast and slow analog signal processing electronics. Five of these "analog boards" are required for each GeD. Each set of five analog boards connects to a common back plane, which supplies bi-directional housekeeping communication, power, and event data channels. Low level input signals connect to the front panel, well away from the back plane and are shielded for noise. Each analog board has two ACTEL Field Programmable Gate Arrays (FPGA). One ACTEL keeps track of the LLD, ULD and fast trigger rates in each strip, while the second coordinates the logic between the slow and fast channels, as well as the shield veto signals. A single Altera NIOS embedded processor board interfaces with each set of five analog boards, and communicates with the main flight computer via an ethernet link. The total power per channel is currently $210 \mathrm{~mW}$, although lower power options are being considered for the $12-\mathrm{GeD}$ instrument.

\subsection{Detector Uniformity}

Since our Compton imaging and background rejection techniques $(8 ; 9)$ rely on reconstructing multiple photon 
interactions across different strips and even detectors, the uniformity between strips and detectors of both the spectral and positioning resolutions is very important. The instrument response has to take into account all of the slight variations between strips, and that is part of our ongoing analysis of the calibration and flight data from the last flight.

In Fig. 7 we show our background "flat field" obtained while at float during the flight. The data has been cut such that only events that triggered one cathode strip and one anode strip are used, and that unique location allows us to plot the events in a pixel display. From this plot one can immediately discern both the curved corners of the detectors as well as the single non functioning strip on detector 0. Other than these artifacts, however, the flat-field detector response is quite uniform over the entire surface of both detectors.

\subsection{D Positioning}

There are two main keys to Compton imaging with this system. The first is to accurately determine the energy deposited in the detector at each interaction. The second is successfully tracking, in all three dimensions, the $\gamma$-ray interactions within the detector. By segmenting the anode into strips, and the cathode into orthogonal strips, 2-D positioning is achieved directly through identification of the active anode and cathode strips.

Measuring the "depth", or " $z$ position", of the interaction in the detector (the distance between the $\gamma$-ray interaction and either the anode or cathode strip), is achieved by measuring the difference between electron and hole collection times on opposite faces of the detector (fig. 8). The Collection Time Difference (CTD) for an event is well defined, and has been shown to be linear with depth to first order $(10 ; 11)$. The drift time across an $15 \mathrm{~mm}$ thick $\mathrm{GeD}$ is on the order of $150 \mathrm{~ns}$ and $200 \mathrm{~ns}$ for electrons and holes, respectively.

To measure the time when the signal on each channel reaches $50 \%$ of its maximum amplitude, we use a simple analog constant fraction discriminator (12) built into each channel's electronics chain. These "fast channels" give us an $0.5 \mathrm{~mm}$ FWHM spatial resolution at $60 \mathrm{keV}$. This is comparable to what get get by digitizing the preamp signals, but with a much smaller, simpler, lower power system.

The calibration of the interaction depth in terms of the CTD poses a unique problem since we cannot send in events of known depth. We can, however, send in events with a known depth distribution. Our technique of converting CTDs to depth, or $z$ position, is discussed in detail in (11) and (13).

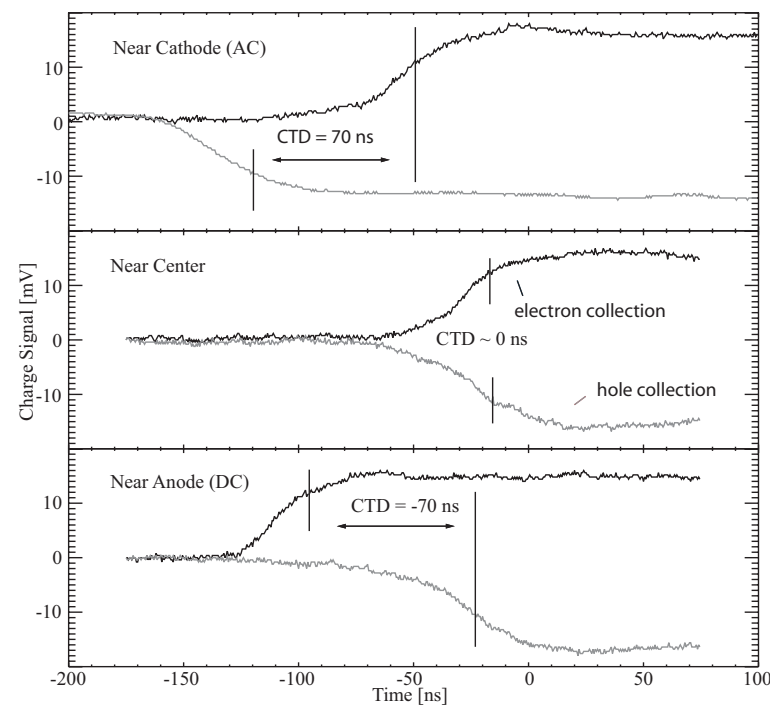

Figure 8. Induced signals on anode and cathode strips of our prototype $\mathrm{GeD}$, for $122 \mathrm{keV}$ photon events near the anode (top), the GeD center (middle), and the cathode (bottom). Our 3-D electronics use a simple delay line to time-tag each strip signal as it crosses $50 \%$ of its maximum amplitude.

\subsection{Spectral Performance}

The spectral resolutions of each of the anode channels taken with the flight electronics are quite good. On average, the the resolutions obtained with the flight electronics are identical to what we get using benchtop electronics. The variation of values obtained with the flight electronics $( \pm 12 \%)$ is also similar to the range seen with lab equipment $( \pm 8 \%)$.

During the flight the entire detector system worked flawlessly. In Figs. 9 and 10 we show representative background strip spectra from both sides of both detectors. As mentioned in $\S 1$, for the prototype flight we oriented the detectors such that detector 0 more faceon than edge-on to the sky, and with detector 1 slightly shielded behind it. That is why the overall intensity of the spectrum from detector 1 is slightly less than detector 0 .

\subsection{Imaging}

With well calibrated positioning and spectroscopy, the NCT and NCT prototype can be used to produce $\gamma$-ray images of source fields with moderate angular resolution. In Fig. 11 show the results of a calibration run of the NCT prototype with a $\left.{ }^{60} \mathrm{Co}(1.173 \mathrm{MeV})\right)$ source placed $\sim 60^{\circ}$ above the instrument pointing axis. The source is obviously evident in the image and is well lo- 

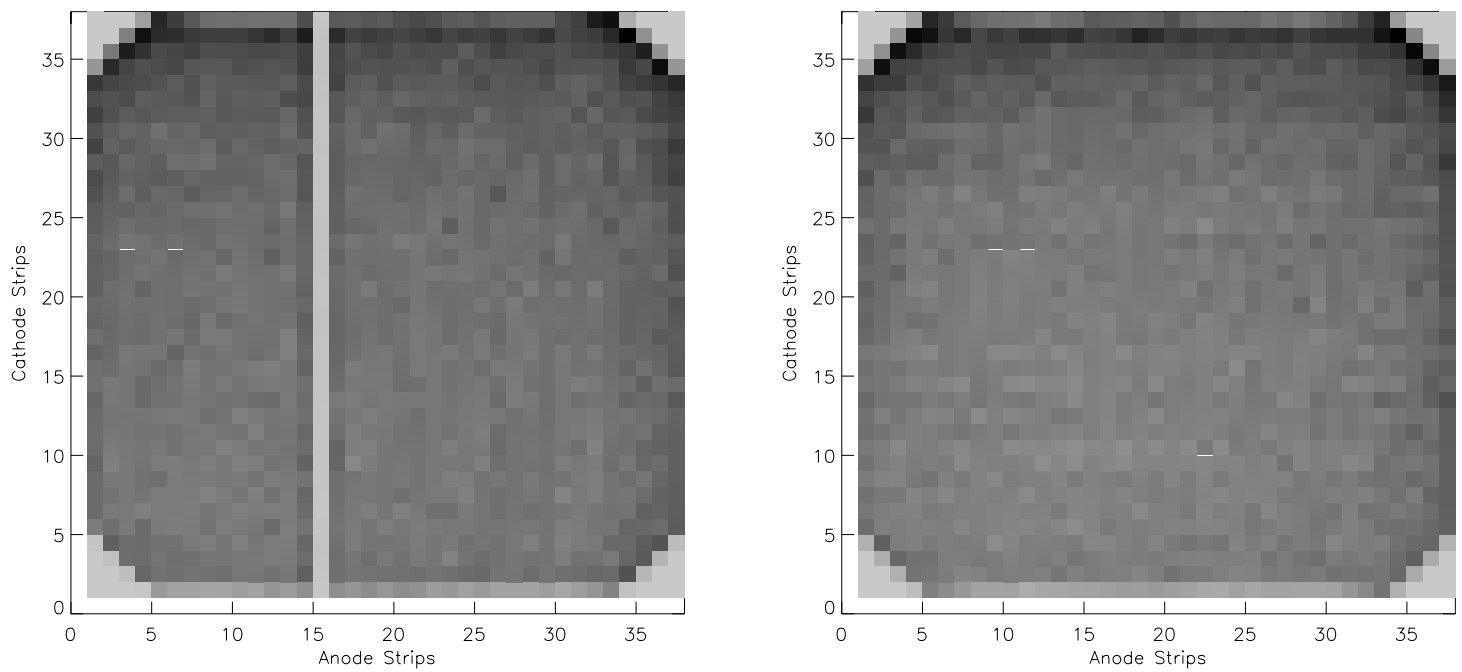

Figure 7. LEFT: detector 0. RIGHT: detector 1. Flat fields of the two detectors obtained while at float during the 2005 balloon flight. The data has been binned as pixels, meaning each event uniquely triggered a single crossed pair of strips. With the exception of strip 15 on detector 0 , which was not functioning for this flight, flat-field detector response is quiet uniform. The curved corners of the detectors can also be seen in these images.
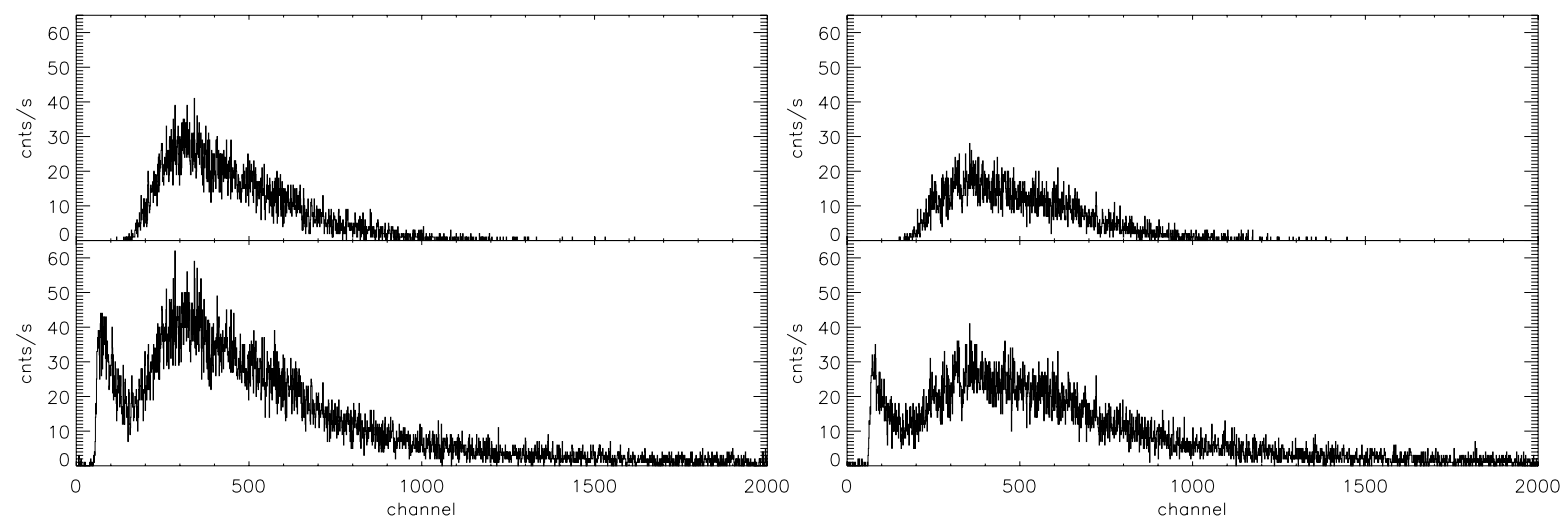

Figure 9. LEFT: Channel spectra of detector 0, anode strip 24 obtained during the 2005 balloon flight. RIGHT: Simultaneous channel spectra of detector 1, anode strip 25. TOP PANELS: Single event spectra, summing only those events when there was no coincident anode event. BOTTOM PANELS: Total strip spectrum, allowing for (but not summing in) events coincident on other anode strips. 

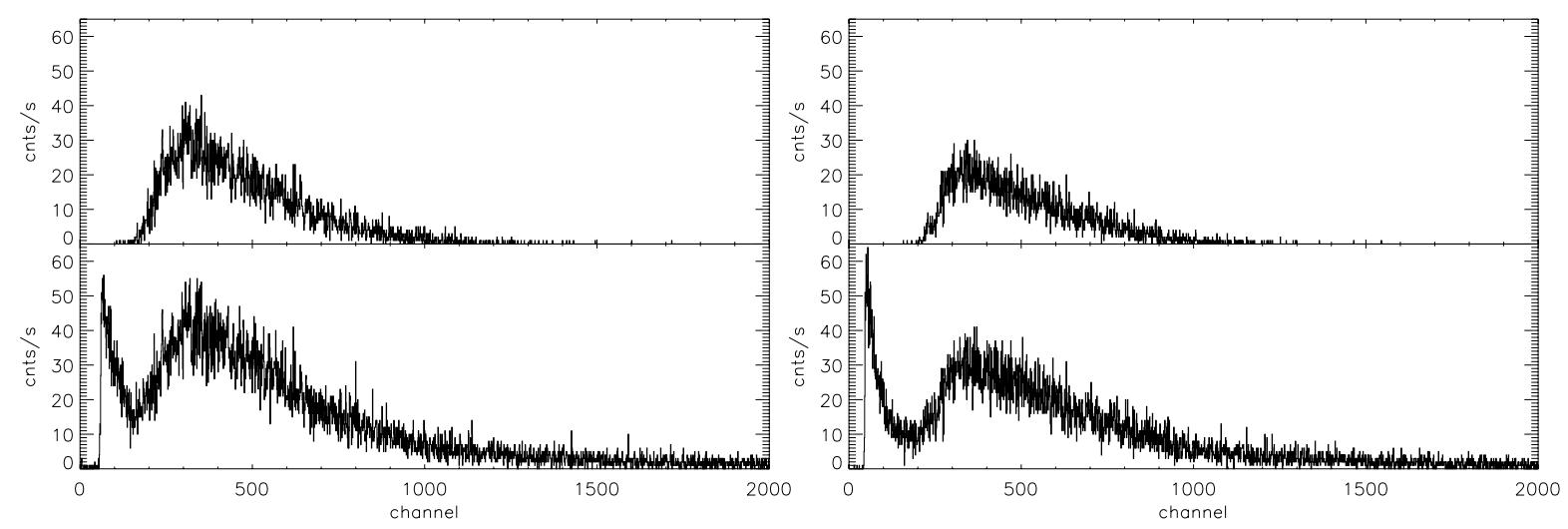

Figure 10. Same plots as in Fig. 9, but using data from cathode strip 25 from each detector.

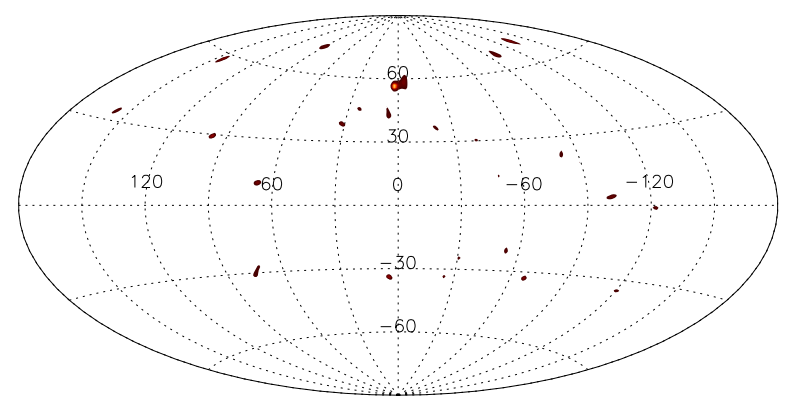

Figure 11. Image of an off-axis ${ }^{60} \mathrm{Co}$ calibration source. The image was produced using XXXX events, and was produced using a standard event list, maximum entropy technique.

calized, demonstrating the wide field of view Compton imaging capabilities of NCT.

\section{GONDOLA SYSTEMS}

The NCT instrument was designed to fit inside the envelope of the existing HIREGS balloon gondola. HIREGS was a LDBF payload that flew four times from Antarctica, the last of which was in 1998 January. Even though the NCT prototype flight was less than twelve hours, our primary goal was to qualify the gondola for a LDBF. Therefore we tried to keep as much of the HIREGS long duration capabilities as possible.

Our detector electronics (excluding our detectors and their preamplifiers), flight computer, data vault, power control unit, and batteries are all contained inside the gondola electronics bay. See Fig. 12 for their relative locations. The electronics bay is a thermal enclosure, designed to maintain the electronics at room temperature. All of the electronics (with the exception of the hard disk drives) are designed to operate in vacuum.

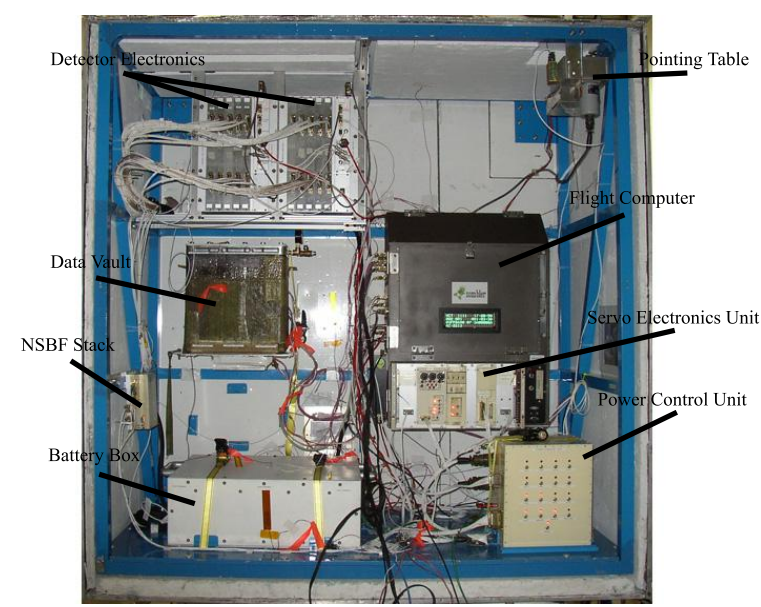

Figure 12. Picture of the gondola electronics bay, showing the relative locations of our various systems.

\subsection{Flight Computer and Data Storage}

The Altera NIOS board in each set of detector electronics interfaces with the flight computer through an ethernet link, and connects through a UDP socket. Since UDP sockets do not handshake, they are considerably faster than TCP sockets and therefore can handle a much higher data rate. The NIOS boards produce four basic packet types labeled according to the information they contain; events, rates, livetimes, and housekeeping.

The flight computer does very little processing of the raw packets other than to add a header, put them in the telemetry, and save them to the data vault. Event packets (1168 bytes) are larger than telemetry packets (160 bytes), so they are split into eight parts, headers are added to each part and they are sent individually. Rate and housekeeping packets have headers added and are sent as is. Livetime packets are collected into groups 
of eighteen, along with a single header, to form telemetry packets.

For telemetry we use a dedicated transmitter, and for the NCT prototype our maximum data rate was $64 \mathrm{kbits} \mathrm{s}^{-1}$. This was more than adequate for our two detector NCT prototype. For NCT we will increase our maximum allowed rate so that we can get full telemetry from all twelve detectors while we are in line of sight with the gondola.

Because a LDBF can last days or even weeks, maintaining a line of sight connection over the entire flight is difficult if not impossible. Therefore we also store each packet in our data vault, regardless of whether or not it was telemetered. For the NCT prototype our data vault was a pair of $32 \mathrm{~Gb}$ SCSI hard disk drives (HDDs), one acting as our primary drive with the second acting as a backup.

Since HDDs do not work in the near-vacuum at float altitude and our sensitive to large shocks and vibrations, our drives were mounted in a spring dampening system and housed inside a small pressure vessel. However, because solid state devices are becoming larger and less expensive, and have the added benefit of working in vacuum and being more robust, we expect to do away with the SCSI drives and pressure vessel for our LDBF.

Our flight computer has an AMD 486DX2, 66 Mhz processor mounted on a Teknor Viper805 single board computer. It boots from an onboard $2 \mathrm{Mb}$ flash drive, and is connected to our various interface cards through a 20-slot EISA backplane. Our flight computer can monitor up to 64 analog inputs (voltages, currents, temperatures, solar sensors, etc.), communicate on up to 10 RS-232 serial lines, and execute up to 48 discrete on/off commands.

\subsection{Pointing}

The flight computer is also responsible for calculating and executing the azimuthal and elevation pointing offsets for the current target, given the current time and gondola position as determined by GPS. The offsets are relative to the horizon in the case of elevation, and either the local magnetic field or current solar position for azimuth. The accuracy of the system is $0.5^{\circ}$ in both azimuth and elevation.

The elevation drive, which was not connected for the NCT prototype but will be for the LDBF, is connected directly to the flight computer through a custom built interface board. To adjust the pointing, the flight computer powers a motor for a calculated amount of time.
The motor is attached to a worm drive, which tilts the entire instrument cradle in elevation. The angle of the cradle with respect to the horizon is checked using a 13bit absolute optical encoder, and the system performs several interations to ensure the correct encoder value is obtained.

Azimuthal pointing is done a little differently. Instead of changing the azimuth pointing directly, the Servo Electronics Unit (SEU), which is built into the flight computer housing, rotates the gondola automatically to null an input sensor. This sensor can either be a solar sensor, causing the gondola to null on the sun, or a magnetometer, in which case the sensor nulls perpendicular to the local magnetic field. Because we wanted the option of an overnight flight for the NCT prototype, we used magnetic pointing instead of solar.

To point at an arbitrary azimuth, the nulling sensor is mounted on the "pointing table." The table assembly has a stepper motor that rotates the sensors, with the angle of rotation again measured by an 13-bit absolute optical encoder. To point in azimuth, the flight computer commands the stepper motor to move a calculated number of steps, again using a custom built interface board, and reads the encoder to check that the position is correct. Once the nulling sensor starts moving, the gondola swings in the opposite direction to keep the servo nulled, thus executing an azimuthal pointing.

An LDBF from Alice Springs, Australia provides an interesting challenge to our current system. Since the gondola will be at float during both day and night, we cannot use solar pointing. Also, the accuracy of magnetic pointing was fine for the prototype flight, but we would like something more accurate for an LDBF. So we are currently looking at options for a new azimuthal pointing system, preferably one based on differential GPS.

\subsection{Power}

The NCT prototype power system is the one system that is going to see the most overhaul between now and the LDBF. For the 2005 flight we used eight nonrecharable LiSO2 batteries, connected to our various computers and electronics though a simplified Power Control Unit (PCU). The PCU provided switched power to the two sets of detector electronics, the flight computer, the azimuth servo motor, and a series of heaters installed in the electronics bay in case the the temperature drops too low. The various power switches were toggled on and off using discrete commands from the flight computer. Running everything 


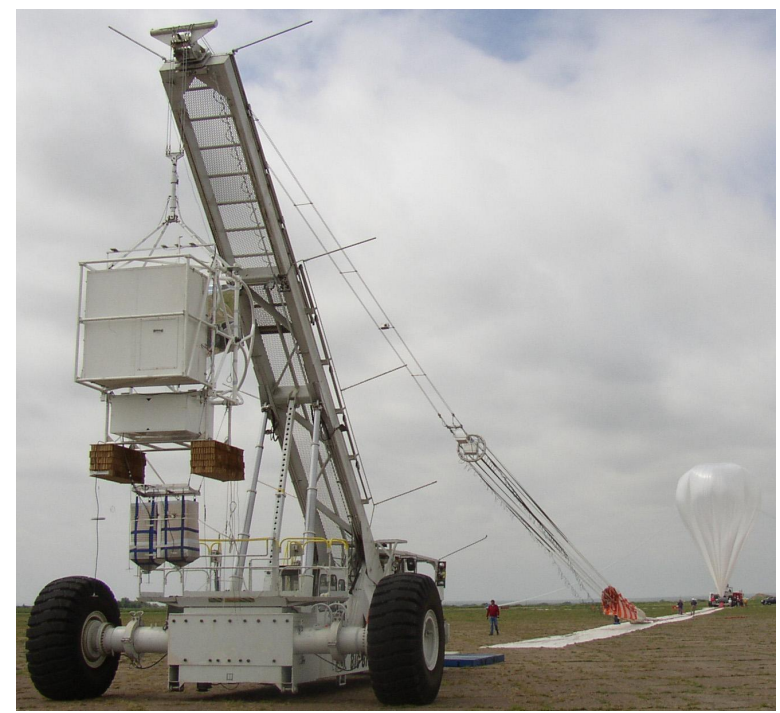

Figure 13. NCT on the flight line, ready for launch.

but the heaters, the 8 batteries were enough to keep the system powered for over 30 hours.

Since the point of an LDBF is to operate at float for longer than 30 hours and we do not want to fly many dozens of batteries, the obvious solution is to use an array of solar panels during the day and batteries at night. This will require adding a set of solar panels and either rebuilding or replacing our current PCU.

\section{2005 FLIGHT}

The NCT prototype was launched on a $39.5 \mathrm{Mft}^{3}$ high altitude balloon from Fort Sumner, New Mexico on 2005 June 1 (Fig. 13). The balloon reached a float altitude of $\sim 133 \mathrm{kft}$ at approximately 20:30 GMT, and was at float for approximately $5.5 \mathrm{~h}$ before termination. Fig. 14 shows the altitude profile as a function of time through the flight, while Fig. 15 shows the flight path.

We had three main goals for this flight. First, we wanted to qualify our gondola for a future LDBF. Second, we wanted to measure the instrumental background, telemetry rates, data vault needs, etc., at float. For the 2005 flight the instrument and associated electronics performed flawlessly, and we achieved both of these goals.

Our tertiary goal was the observation of two science targets, the Crab Nebula/Pulsar and the transient accreting pulsar $1 \mathrm{~A} 0535+262$. Both pulsars are spatially close on the sky, separated by $\sim 4^{\circ}$, and therefore simultaneously fell with within the NCT field of view. The Crab was chosen because it is a well studied source, and an excellent one to calibrate on. The

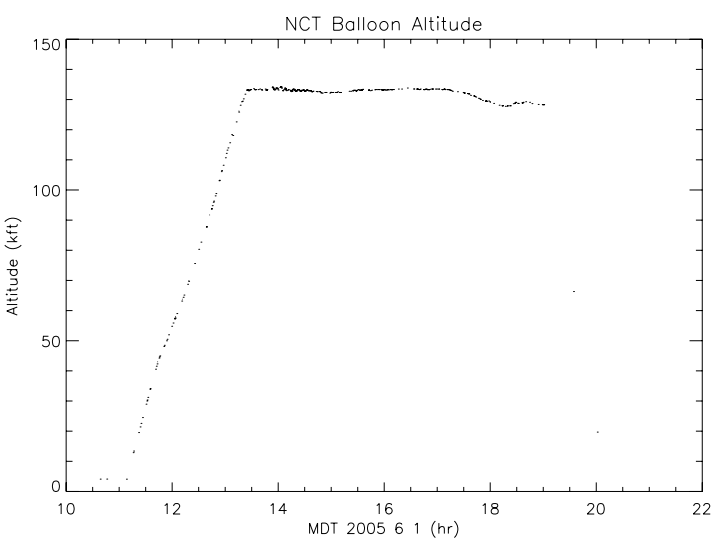

Figure 14. Altitude of the NCT balloon gondola for the 2005 June 1 flight. The entire flight lasted approximately nine house, $\sim 5.5 \mathrm{~h}$ of which were at a nominal altitude of $133 \mathrm{kft}$.

pulsar $1 \mathrm{~A} 0535+262$ is transient, and we were fortunate enough that it was in a rare outburst state during our flight. In addition to being able to image two bright point sources, 1A0535+262 provided the opportunity to search for hard X-ray cyclotron absorption features with unprecedented spectral resolution.

As discussed in detail above, the gondola pointing system was set up to keep the instrument at a fixed elevation, but to actively point in azimuth to keep the Crab centered in the field of view. Unfortunately, shortly after launch the azimuthal pointing system failed, seriously compromising our exposure to our sources and adding an extra level of complexity to the data analysis. The data analysis project is ongoing, and right now we are focusing on calibration (3) and detector background (4).

Our balloon gondola frame suffered some minor and mostly cosmetic damage during impact, but is otherwise fine. The NSBF parachute termination package worked flawlessly, releasing the parachute from the gondola right after landing. Since the flight, the entire detector and data system has been powered up in the laboratory and is working fine. Neither the detectors, cryostat, nor electronics suffered any damage during the flight, landing, or recovery.

The most important result of the flight, however, was the fact that the instrument and associated electronics functioned flawlessly for the duration. As discussed previously, in Figs. 9 and 10 we show representative anode strip spectra obtained while at float altitude. The average total count rates in detectors 0 and 1 were $43.4 \pm 7.5 \mathrm{cnts} \mathrm{s}^{-1}$ and $38.5 \pm 9.5 \mathrm{cnts} \mathrm{s}^{-1}$ respectively, 


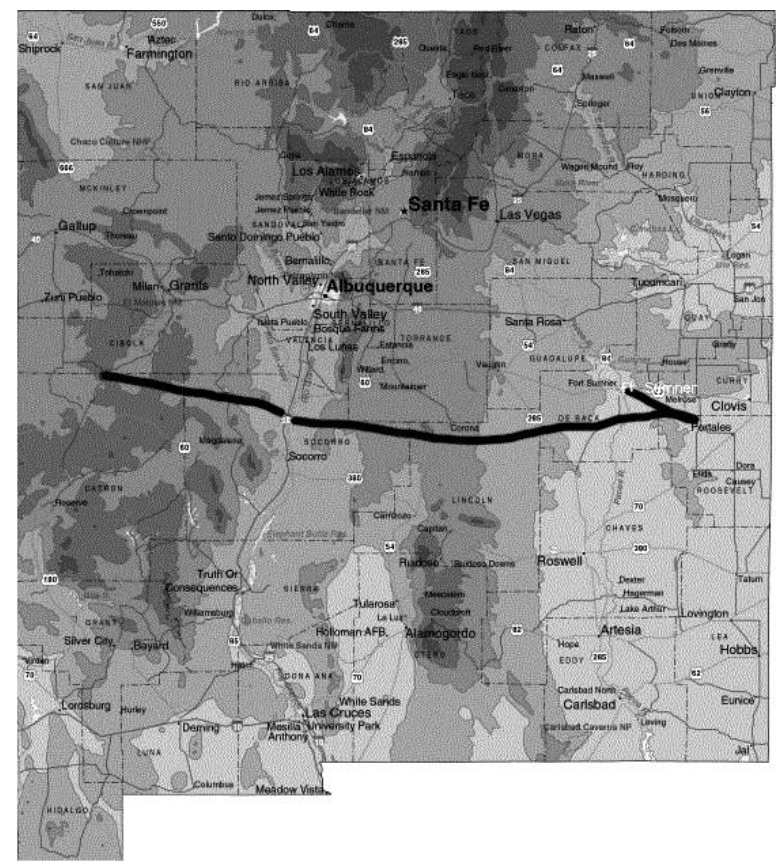

Figure 15. The NCT balloon flight path for the 2005 June 1 flight, shown as a heavy black line. On ascent the gondola initially moved eastward, but quickly started heading west at $30-35$ knots. The flight was terminated over western New Mexico shortly before local sunset.

well below our predicted background rates. Overall the system logged more than 2.3 million events while at float. The detectors and shields worked well, our detector electronics had no problems handing the incoming data, and all strips and channels performed as expected. So, although there was a failure in the pointing system and the data analysis is still ongoing, we are confident that this flight will be a critical step in demonstrating the viability of the NCT concept for future space missions such as the Advanced Compton Telescope.

\section{FUTURE WORK}

Our next step is to prepare the instrument and gondola for a long duration balloon flight from Alice Springs, Australia in the fall of 2007. For the next flight, we plan on fabricating four more GeDs and integrating them into our existing cryostat. This will being our total number of detectors to six. The detector material is currently at LBNL to be processed. We also need to build and associated four more sets of detector electronics, which should go quickly now that the design has been proven in flight. The gondola needs some minor mechanical work, but overall is in excellent shape. We are planning a redesign of our azimuthal pointing system, both to fix the failure and to incorporate differential GPS for positioning.

\section{ACKNOWLEDGMENTS}

The work was supported by NASA Grant NAG5-5350

\section{References}

[1] S. E. Boggs et al. in Gamma 2001, AIP Conf. Proc., 587, p. 877, 2001.

[2] S. E. Boggs et al. in IEEE NSS Conf. Proc., N14-5, 2001.

[3] M. E. Bandstra et al., 2005. in preparation.

[4] J. D. Bowen et al., 2005. in preparation.

[5] P. N. Luke et al. in IEEE Nucl. Sci. Symp. Conf., 39, p. 590, 1992.

[6] L. Fabris, N. Madden, and H. Yaver NIM, p. A424, 1999.

[7] L. Fabris, N. Madden, and H. Yaver NIM A424, p. 545, 1999.

[8] S. E. Boggs and P. Jean $A \xi A S$ 145, p. 311, August 2000.

[9] S. E. Boggs and P. Jean $A \xi A$ 376, p. 1126, September 2001.

[10] M. Amman and P. N. Luke NIM A452, p. $155,2000$.

[11] S. Amrose et al. in IEEE NSS Conf. Proc., N12-22, 2001.

[12] M. T. Burks et al. in IEEE Nuclear Science Symposium, November 2001.

[13] W. Coburn et al. SPIE 4784, p. 54, 2002. 